\title{
KILLING THE ELECTRON CLOUD EFFECT IN THE LHC ARCS*
}

\author{
N. Diaczenko, A. Jaisle, P. McIntyre, N. Pogue \\ Dept. of Physics, Texas A\&M University, College Station, TX 77843 U.S.A.
}

\begin{abstract}
A getter/electrode assembly has been devised to suppress the regeneration mechanism of the electron cloud effect in the arc dipoles of LHC. The assembly consists of a copper foil electrode, supported through insulators on a stainless steel skid. The assembly is attached to the bottom surface of the beam screen using BeCu hairpins. The top surface of the $\mathrm{Cu}$ electrode can be coated with nonevaporative getter (NEG) to provide distributed pumping of all non-inert gases within the beam screen. When the electrode is biased $+100 \mathrm{~V}$, electrons from beam ionizations and photoionization are cleared in $<25 \mathrm{~ns}$, killing the regeneration mechanism for any feasible bunch spacing. The NEG surface can be regenerated by passing a current through the electrode to heat it to $\sim 240 \mathrm{C}$. The heat transfer (radiant + conductive) to the beam screen during regeneration is small enough so that the beam screen could be maintained at nominal $20 \mathrm{~K}$ temperature during regeneration.
\end{abstract}

\section{ELECTRON CLOUD EFFECT}

The electron cloud effect (ECE) arises from the multipactoring of electrons within the vacuum chamber. In the Large Hadron Collider (LHC) electrons are produced when gas molecules are ionized by protons in a highintensity bunch (dominant at injection energy), and also when synchrotron light strikes the beam screen (dominant at collision energy). The critical energy of synchrotron light at collision energy is $44 \mathrm{eV}$, for which photoemission yield from copper is maximum.

Such electrons typically have $\sim \mathrm{eV}$ energies and so are still inside the beam screen when the next proton bunch passes. The Coulomb field of that next bunch is sufficient to accelerate ambient electrons to energies of $\sim 100-1000$ $\mathrm{eV}$. After the bunch passes each such energetic electron travels to the side wall before arrival of the next bunch, strikes the wall, yielding one or more secondary electrons.

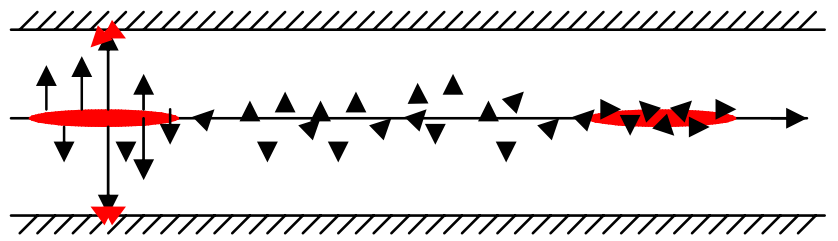

Figure 1. Schematic illustration of electron cloud effect. First bunch produces slow electrons, fields of second bunch accelerate residual electrons to produce secondary emission.

*Work supported by US Dept. of Energy, grant \# DE-FG03-95ER40924, and by EC Research Infrastructure Activity FP6: 'Structuring the European Research Area’ program, CARE-RII3-CT-3003-506395.

\#p-mcintyre@physics.tamu.edu
If the secondary electron yield $\delta>1$, the process is regenerative and the ambient electron density will grow exponentially. For copper surfaces such as the beam screen, $\delta$ typically has values ranging from a low of $\sim 1.1$ to a high of $\sim 1.7$. The value of this parameter, notoriously difficult to control, is thus critical to the multipactoring process of the electron cloud effect.

ECE has several bad effects on collider operation. First, the electron distribution responds dynamically to the proton bunch as it passes and can excite transverse modecoupling instability (TMCI), coupled-bunch instabilities, head-tail motion within the proton bunch, tune spread, beam loss and incoherent emittance growth. There is an extensive literature on the effect of electron cloud effect on beam dynamics [1]. Zimmermann and Benedetto give a recent summary of the understanding [2]. Simulations using the HEAD-TAIL code indicate the possibility of long-term emittance growth that could be detrimental with a storage time of hours. Also transverse mode-coupling instabilities will be excited by the electron cloud. While it has been possible to control fast instabilities in the SPS, control has required a degree of chromaticity that could be problematic for LHC operation. Presently there is no cure for long-term incoherent emittance growth due to the electron cloud, other than reducing the electron density.

Second, the electron cloud desorbs gases from the walls of the beam screen, and could push the limit for beam lifetime and pressure-bump instabilities. It has been proposed that the surfaces of the beam screen could be conditioned. Conditioning appeared to improve the pressure in the beam screen in the COLDEX experiment [3], but the heat load attributed to ECE did not reduce with time.

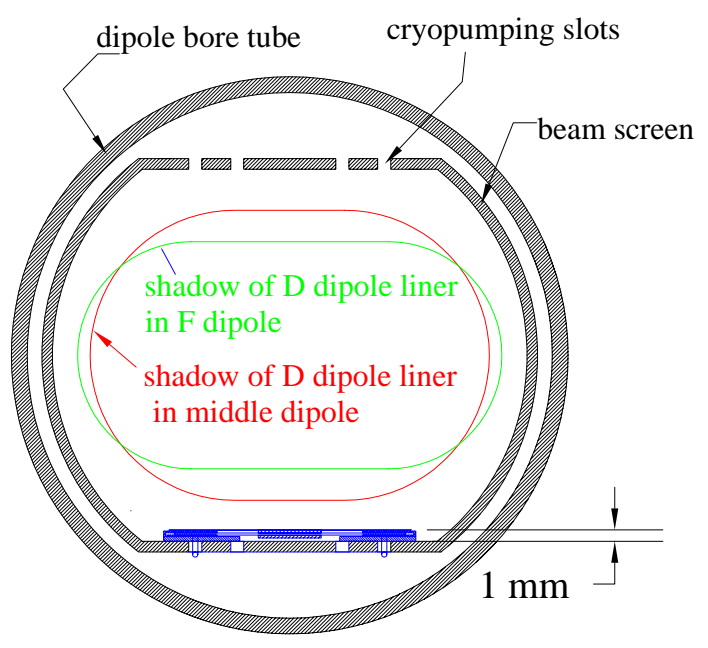

Figure 2. ECE electrode assembly (blue) on base of beam screen. Shadows of the beam screen from the D dipole are shown at the F dipole (green) and middle dipole (red). 


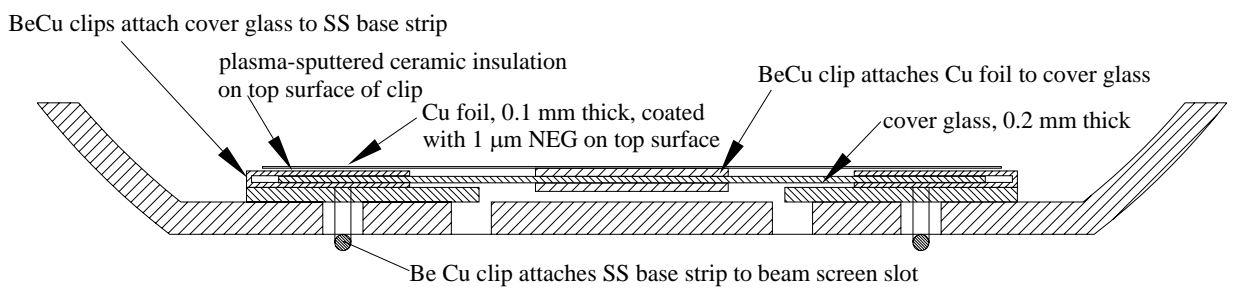

Figure 3. Detail of ECE electrode assembly.

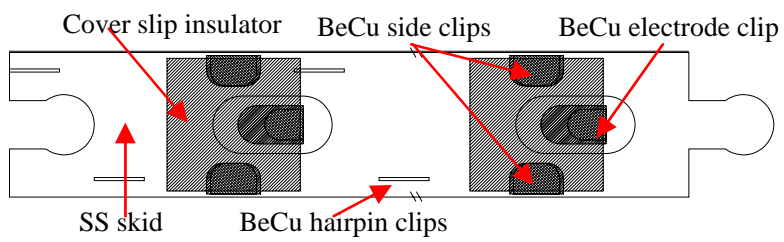

Figure 4. Plan view of a segment of the ECE electrode, showing attachement of $\mathrm{Cu}$ electrode to SS skid.

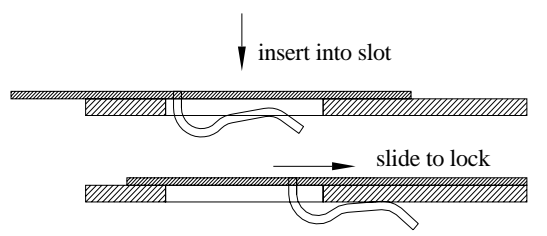

Figure 5. Detail showing attachment of SS skid through slots in beam screen.

Lastly the energetic electrons heat the surfaces that they impact. This heat has been studied both by models and by experiment [4], and is expected to be $\sim 1-2 \mathrm{~W} / \mathrm{m}$ for LHC bunch intensity, depending upon the value of $\delta$ for the copper surfaces of the beam screen. The heat load from ECE with bunches of $10^{11}$ protons could exceed installed refrigeration capacity for $25 \mathrm{~ns}$ bunch spacing (the mode most favorable for high-luminosity physics), and might limit operation to the 75 ns bunch spacing foreseen for initial operation. A shield has been installed behind the slots that couple the beam screen vacuum to the cold walls in order to reduce the transport of these energetic electrons onto the cold bore. Unfortunately it also reduces cryopumping by a factor 3 .

\section{THE ECE ELECTRODE}

The ECE electrode is a getter/electrode structure designed to integrate into the beam screen as shown in Figure 4. It contains a copper electrode, mounted on a 316LN stainless steel skid which is in turn anchored to the floor of the beam screen. The electrode is electrically and thermally isolated to operate at $+100 \mathrm{~V}$ with respect to the beam screen.

The electrode can be coated with $\mathrm{Ti} / \mathrm{Zr} / \mathrm{V}$ getter following the process developed by Benvenuti et al. [5]. A coating of a $\sim 2 \mu \mathrm{m}$ thick NEG layer on the electrode could be performed in the magnetron sputtering facility of Chiggiato at CERN. The NEG layer would provide distributed pumping within the beam screen to assist the cryopumping to the bore tube in the arcs.

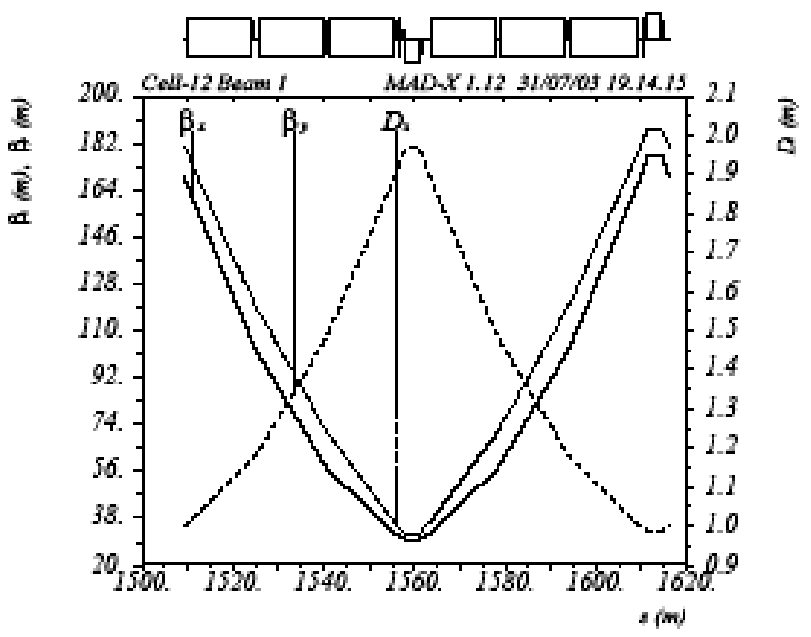

Figure 6. Arc lattice, showing $b_{v}$ in the three dipoles of a half-cell.

The electrode is insulated from the skid by means of a thin glass plate (a standard $22 \mathrm{~mm}$ square microscope cover glass). The borosilicate glass has large but finite resistivity, so that any charge developed on the insulator during operation would be cleared. The copper electrode is attached to the glass insulator by a $\mathrm{BeCu}$ tab clip; the glass is attached to the skid by two $\mathrm{BeCu}$ side tab clips as shown in Figure 4.

It is necessary to provide sufficient thermal isolation to limit heat transfer to the beam screen so that the electrode can be heated to $240 \mathrm{C}$ to activate the NEG layer without increasing the beam screen temperature. The arrangement shown does this by providing a $\sim 1 \mathrm{~cm}$ long conduction path through the $0.2 \mathrm{~mm}$ thick glass from the skid clip to the copper clip.

The copper foil electrode is chosen to be $125 \mu \mathrm{m}$ thick, sufficient to provide a low impedance for image currents of the proton beam but thin enough to provide a favorable characteristic for heating the strip during NEG regeneration. The top surface of the side tab clips is coated with a plasma-sprayed $\mathrm{Al}_{2} \mathrm{O}_{3}$ insulating layer to provide reliable electrical isolation from ground.

The skid is fabricated from six $2.5 \mathrm{~m}$ segments of straight 316LN material. The ends of each segment are die-cut to form a key-in-lock joint to the next segment as shown in Figure 4. The curvature required for the $9 \mathrm{~mm}$ magnet sagitta of the dipole is accommodated in the keyin-lock joints. The joints are spot-welded to lock them together. The foil electrode is sufficiently flexible that it can be fabricated straight and mounted to the slightly 
curved skid. The pattern of hairpin clips has been designed so that the 6 segments can be assembled and trimmed to make an exact fit to the pattern of slots in each individual dipole, even though the registration of that pattern is different for each dipole.

During cooldown the copper electrode will shrink by a strain $\delta \mathrm{L} / \mathrm{L}=3 \times 10^{-4}$ relative to the SS skid. The differential shrinkage is accommodated by bowing the electrode slightly as it is attached at the successive insulators; the additional length is controlled by rod spacers placed between the skid and the foil electrode midway between the locations of successive mounting tabs. Once the tabs are all attached to the insulators the spacers are removed.

\section{APERTURE CONSIDERATIONS}

The electrode assembly occupies $1.2 \mathrm{~mm}$ of vertical aperture, as shown in Figure 2. In the three dipoles of a given half-cell of the arc lattice, the vertical betatron function $\beta_{\mathrm{v}}$ has different maxima in each of the three dipoles, as shown in Figure 6 The dipole nearest the horizontally focusing quadrupole presents the most constraining vertical aperture. If one maps that constraining aperture with $\beta_{\mathrm{h}}, \beta_{\mathrm{v}}$, and dispersion $\mathrm{D}$, the 'shadow' of the $\mathrm{D}$ dipole at the other two dipoles are shown in Figure 2. If the electrode assembly were installed in only those two of the three dipoles in every half-cell, there would be no reduction in beam aperture at all. If the electrode assembly were installed in the third dipole, vertical aperture would be reduced by $1.2 \mathrm{~mm}$.

\section{BEHAVIOR DURING MAGNET QUENCH}

During magnet quench, the current in a dipole decreases from full excitation to zero in $\sim 0.1 \mathrm{~s}$. The timechanging flux through the beam screen region induces currents in the copper lining of the screen and also in the electrode proposed here. Rathgen [6] showed that while induced currents primarily cause tension within a conducting structure within a uniform-field dipole, the small multipole fields produce some net forces as well. In order to evaluate this possibility for the ECE electrode assembly, we simulated the quench in the body of an LHC dipole, including the allowed field multipoles, using the 2-D electromagnetic code PE2D [7].

The outcome of these calculations is as follows:

- The electrode will dissipate $\sim 550 \mathrm{~J} / \mathrm{m}$, heating it to $\sim 160 \mathrm{~K}$.

- The electrode will experience $\sim 220 \mathrm{MPa}$ of balanced tension, which is less than half the yield strength of the cryogenic copper.

- The maximum instantaneous unbalanced force acting on the electrode consists of

o $20 \mathrm{~N} / \mathrm{m}$ horizontal (due to quadrupole);

o $170 \mathrm{~N} / \mathrm{m}$ vertical (due to sextupole).

- That force loading is well within the strength of the $\mathrm{BeCu}$ clips attaching the electrode, the insulator, and the skid.
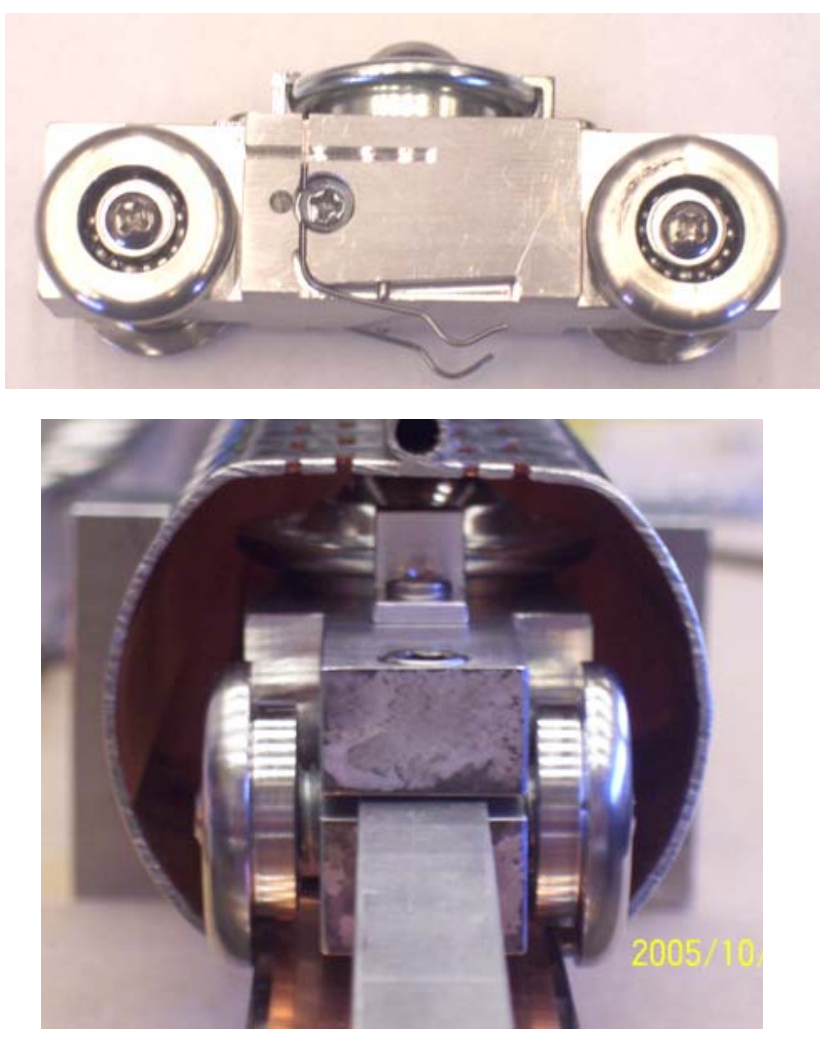

Figure 7. Installation procedure: a) trolley car; b) insertion of trolley train into beam screen.

- The vertical force would deflect the two outer edges of the electrode upwards. The deflection has been modeled using ALGOR [8]; the largest deflection is $.03 \mathrm{~mm}$.

If the electrode were to extend into the end region of the dipole, the non-uniform fields there would produce much larger unbalanced forces and could destroy the structure. For this reason the electrode is terminated $\sim 0.5$ $\mathrm{m}$ into each end of the dipole so that it is completely contained within the uniform-field region of the dipole.

\section{IMPEDANCE}

The impedance of the beam screen is important for longitudinal instability mechanisms. The copper surface of the ECE electrode provides the same impedance for image currents as does the beam screen itself. It will be important to terminate the electrode in such a way that image currents launch from the bottom of the beam screen onto the electrode at one end and then off at the other end with high-capacitance coupling.

\section{ASSEMBLY IN DIPOLE}

Because the ECE electrode has only recently been devised, it will be necessary to install the assembly into each beam screen after it is already installed in a dipole. For this purpose a trolley arrangement has been devised: a string of 50 cars as shown in Figure 7. First the complete electrode assembly is inserted in the dipole on a carrier tray, located to the correct position, and the carrier is re- 


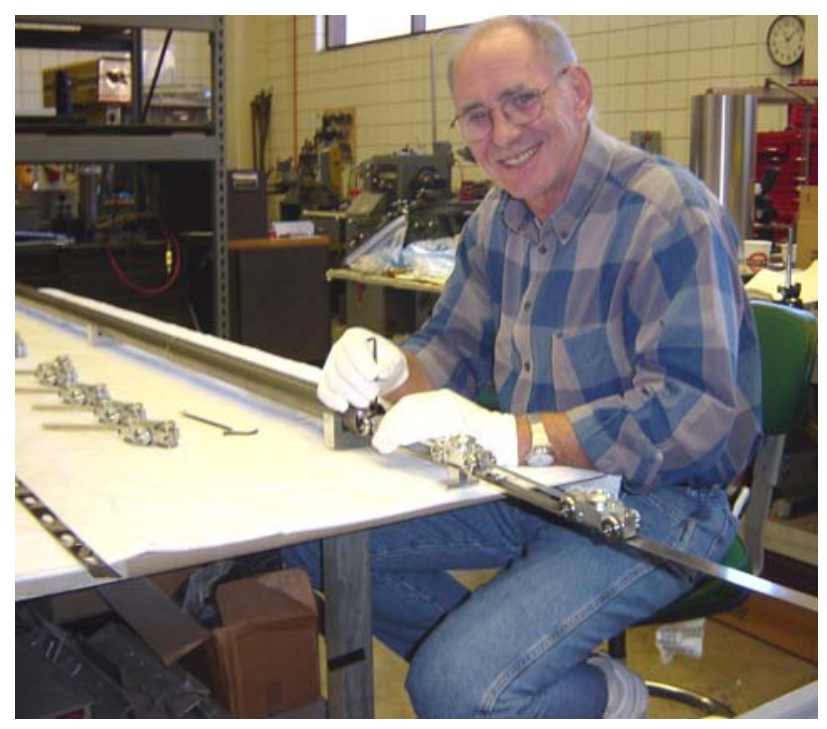

Figure 8. Installation of a prototype ECE assembly in a $3 \mathrm{~m}$ section of LHC beam screen.

moved. The lead trolley car is then positioned so that it sits on the near end of the assembly and locates itself in the beam screen from the bottom corners and a springloaded idler bearing against the top. As the train is pulled through the length of the dipole, the wheel assemblies maintain local alignment within the beam screen and a set of spring fingers on each car (Figure 7a) aligns the electrode assembly and presses it down so that the spring clips are positioned in the beam screen slots (Figure 5a).

As each car proceeds, another is attached behind it so that finally the entire electrode assembly is in the proper position. Then the ECE assembly is pulled towards the lead end to lock the clips to the screen (Figure 5b). This 'terrain-following' design assures that the ECE electrode can be locally anchored to the beam screen even as it curves with the dipole sagitta and sags slightly between dipole supports. Figure 8 shows the installation of the ECE within a $3 \mathrm{~m}$ section of LHC beam screen. Figure 9 shows the completed ECE within the beam screen.

\section{CONCLUSIONS}

An electrode/getter assembly has been designed that is capable of being installed in the LHC arc dipoles in vertical aperture of $1 \mathrm{~mm}$ along the bottom of the beam screen. The electrode should clear all electrons and eliminate the electron cloud effect in the arcs. If the electrode surface is coated with a NEG layer it would provide distributed pumping within the beam screen. As a collateral benefit, the electron current collected on each electrode will give a direct measurement of the beam vacuum in that dipole, which information is not otherwise available and should be very useful during commissioning and operation of the collider.

A full-length prototype ECE electrode assembly has been completed and will be installed during Fall 2005. Pending evaluation of the installation procedure and logis-

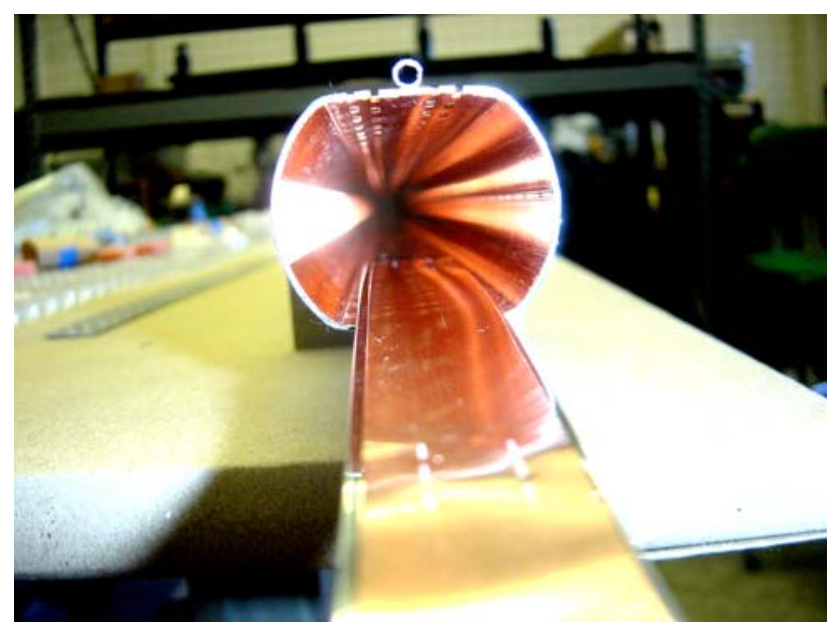

Figure 9. ECE assembly anchored to the bottom of the LHC beam screen.

tics, it could be available for installation in dipoles prior to installation in the LHC arcs.

\section{ACKNOWLEDGMENTS}

It is a pleasure to acknowledge the help and encouragement of Paolo Chiggiato, Lucio Rossi, and Davide Tommasini (CERN), and discussions with Frank Zimmermann and Francesco Ruggiero (CERN) and Miguel Furman (LBNL) on the role of ECE in LHC beam dynamics. The development of prototype ECE assembly was funded by a grant from the George P. and Cynthia W. Mitchell Foundation.

\section{REFERENCES}

[1] http://wwwslap.cern.ch/collective/electron-cloud/

[2] F. Zimmermann and E. Benedetto, 'Electron cloud effects in the LHC', ICFA Newsletter 32 (2004).

[3] J.M. Jimenez et al., LHC Project Report 677 (2003).

[4] V. Baglin and B. Jenninger, Phys. Rev. ST Accel. Beams 6, 063201 (2003).

[5] C. Benvenuti et al., Vacuum 60, 57 (2001).

C. Benvenuti et al., Vacuum 71, 307 (2003).

[6] C. Rathgen, LHC Project Report 582 (2002).

[7] http://www.vectorfields.com

[8] http://www.algor.com 